\title{
Using redundancy to strengthen the relaxation for the global optimization of MINLP
}

\author{
Juan P. Ruiz and Ignacio E. Grossmann* \\ Department of Chemical Engineering \\ Carnegie Mellon University \\ Pittsburgh, PA 15213
}

\begin{abstract}
In this paper we present a strategy to improve the relaxation for the global optimization of nonconvex MINLPs. The main idea consists in recognizing that each constraint or set of constraints has a meaning that comes from the physical interpretation of the problem. When these constraints are relaxed part of this meaning is lost. Adding redundant constraints that recover that physical meaning strengthens the relaxation. We propose a methodology to find such redundant constraints based on engineering knowledge and physical insight.
\end{abstract}

Keywords: Global Optimization, Reduction Constraints, Tight Formulations

\section{Introduction}

Mixed-integer Nonlinear Programming (MINLP) has been widely used in the Process Systems area to represent discrete and continuous optimization problems [1]. In general, the continuous relaxation of these problems are nonconvex. As a result, the direct application of algorithms such as Generalized Benders Decomposition (GBD) [2] [3], Outer Approximation (OA) [4], Extended Cutting Plane [14], may fail to find the global optimum since the solution of the NLP subproblem may correspond to a local optimum and the cuts in the master problem may not be valid. Therefore, specialized algorithms should be used to find the global optimum [5] [6] [7]. In the last decade, many global optimization algorithms have been proposed. However, most of them can be regarded as some particular implementation of the spatial branch and bound framework [6] [8]. The efficiency of these methods heavily relies on having tight relaxations and

\footnotetext{
${ }^{*}$ Corresponding author. Tel.: +1-412-268-2230; fax:+1-412-268-7139; e-mail: grossmann@cmu.edu (I.E. Grossmann)
} 
that is why many of the contributions in this area have been related to this subject. Most of the current approaches to obtain convex relaxations for nonconvex problems are based on the use of predefined convex envelopes [5], leading to formulations where some of the physical meaning is lost. For example, physical laws like mass and energy balances may not be satisfied. Hence, adding constraints that are redundant in the original formulation and which enforce such laws while keeping the program convex, can strengthen the relaxation. Redundancy is usually not considered a good practice in process modeling. However, we show that this often has a great impact in the tightness of the final relaxation in nonconvex problems without incurring in significant increase in the problem size. The explanation behind this behavior can be obtained by realizing that even when alternative nonconvex representations may contain the same information (i.e. keep the same physical meaning), their relaxations do not.

Sherali and Alameddine [9] proposed the RLT procedure to improve the representation of the problem when it is relaxed through the generation of redundant constraints in bilinear nonconvex programs. This is accomplished by using pairwise products of the linear inequalities originally present, previous to their linearization. Based on a similar idea Liberti [10] and Liberti and Pantelides [11] proposed a method using reduction constraints, which improves the efficiency of the implementation. However, for the general case, the problem of systematically finding useful redundant constraints remains a major challenge. Revisiting the physical meaning behind the problem may still be required. Previous results in this respect were reported by Quesada and Grossmann [12] in process networks design and Tawarmalani and Sahinidis [5] in the p-q pooling problem. However, to the best of our knowledge, no systematic methodology based on this approach for more general nonconvex problems has been proposed.

It is the aim of this work to formalize and extend the idea outlined above to any nonconvex MINLP problem whose physical meaning is well defined. The paper is organized as follows. In section 2 we present the theoretical framework of the methodology proposed. We recognize that for a given problem many formulations can be developed based on engineering knowledge and physicial insight. The combination of these formulations give rise to a composite model whose relaxation proves to dominate the relaxation of any of the individual formulations. This idea is illustrated in section 3 . In section 4 we use the theory developed in section 2 to come up with an efficient implementation of the method giving rise to a set of strong cuts (i.e. Generalized Reduction Constraints) that can be added to the original formulation to strengthen its relaxation. We show with a number of examples how these cuts can be obtained from the composite model. 
In section 5 we present a set of examples to illustrate the application of the methodology. Finally, in section 6 we compare the proposed relaxation with the ones obtained from traditional modeling approaches that rely on the selection of a single monolithic nonconvex model.

\section{Theoretical Framework}

The main philosophy of this methodology lies on viewing the relaxation of a nonconvex problem as a process in which some of its physical meaning is lost rather than just a mathematical reformulation that aims at finding a convex set that contains the original feasible region. As we argue in this section, this different way of looking at relaxations, naturally leads to a general framework that can be used to obtain tighter relaxations for nonconvex problems.

The first step before trying to find the optimal solution of a problem consists of understanding its physical meaning from which a high abstraction level representation (HALR) can be generated. This representation is nothing but a collection of all the assumptions, parameters, physical laws that describe the problem accurately. As a second step, a mathematical program (often nonconvex) is developed which captures all the information contained in (HALR). Finally, in order to globally optimize the problem using a spatial branch and bound framework, a relaxation is produced. It is in this step, where the physical meaning, ultimately held in the original (HALR), is lost. In Figure 1 the information flow from the higher abstraction level representation of a problem to its relaxation is presented.

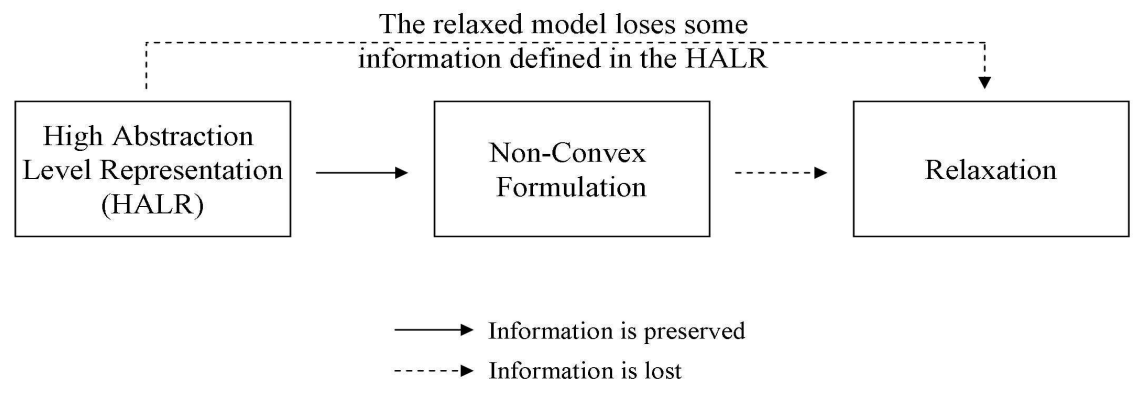

Figure 1: Information flow in modeling and relaxation

Traditional approaches find a relaxation by replacing the nonconvex functions with convex over and underestimators. In order to do so, different strate- 
gies that take advantage of the particular nature of the functions have been proposed. For example, McCormick [13] proposed to replace nonconvex bilinear functions defined in a bounded domain by a set of four half spaces leading to a linear, hence convex, relaxation. In the last years, methodologies for more general set of functions have been presented [5] [19]. The strength of the relaxations will, of course, depend on what specific technique is used for the relaxation and that is why a lot of effort is put on improving these techniques. For instance, Sherali and Alamedine [9] exploited the interaction of the constraints in the original formulation for deriving tighter relaxations using the RLT procedure, while Liberti [10] and Liberti and Pantelides [11] proposed an alternative method to find these redundant constraints more efficiently. Even though the last two approaches mentioned improve the relaxations, the way they generate cuts is based on the use of the nonconvex formulation. In this work we will show how to use instead the (HALR) to generate useful cuts.

A more accurate representation of the information flow diagram is given in Figure 2 .

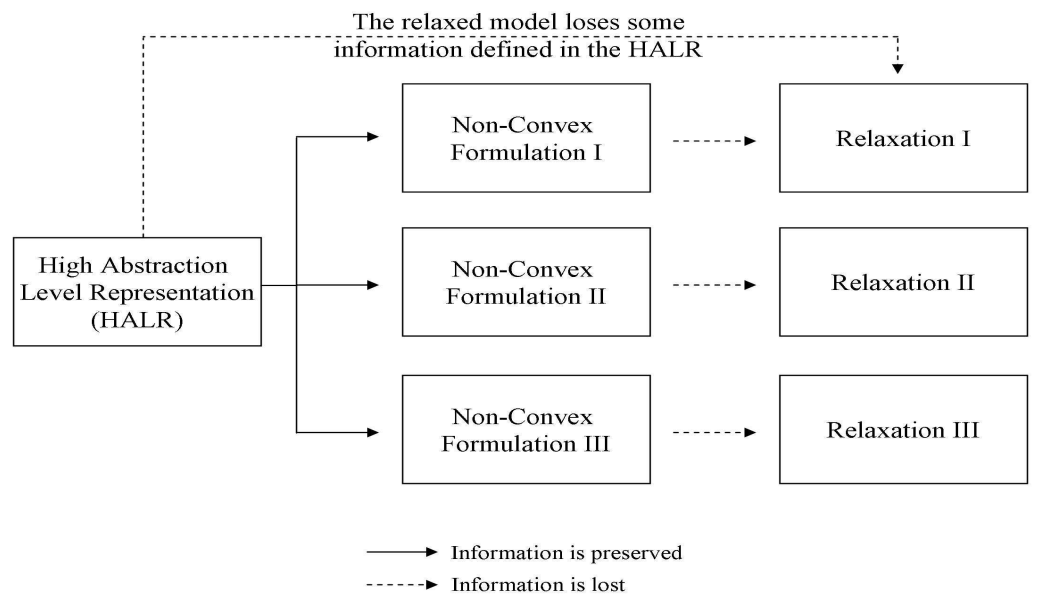

Figure 2: Information flow with multiple formulations

Note that for a given (HALR) based on engineering knowledge and physical insight, a plethora of different nonconvex formulations can be developed. However, it is easy to show that during the relaxation different portions of the physical meaning are lost, ultimately leading to different relaxations. Moreover, 
as proved in the theorem below, these relaxations are often not dominated by the others. As a result, a model that combines all these formulations will lead to a stronger relaxation. We call this representation, composite model. The following theorem, which formalizes this idea, is crucial in the development of this work.

Theorem 2.1 Given a problem in (HALR) form and a set of valid alternative formulations $F_{n}, n \in N$, with relaxations $\operatorname{Rel}\left(F_{n}\right), n \in N$, we can generate a valid formulation $F$ (i.e. composite model) by intersecting $F_{n}, \forall n \in N$, leading to a relaxation $\operatorname{Rel}(F)$ such that:

$$
\operatorname{Rel}(F) \subseteq \operatorname{Rel}\left(F_{n}\right) \quad \forall n \in N
$$

Proof Let us consider the (HALR) form with $|N|$ possible formulations where the formulation $F_{n}$ for a given $n \in N$ is described as:

$$
\begin{array}{cl}
\min & Z=f(x) \\
\text { s.t. } & g(x) \leq 0 \\
& h_{n}(x) \leq 0 \\
& x^{l o} \leq x \leq x^{u p}
\end{array}
$$

where $f: R^{k} \rightarrow R^{1}, g: R^{k} \rightarrow R^{m}$ and $h_{n}: R^{k} \rightarrow R^{p n}, n \in N$, a subset of the variable $x$ are discrete and $h_{n}(x) \leq 0$ is the set of constraints that only appear in formulation $\mathrm{F}_{n}$.

We can generate a formulation $F$ for (HALR) as follows:

$$
\begin{array}{cl}
\min & Z=f(x) \\
\text { s.t. } & g(x) \leq 0 \\
& h_{n}(x) \leq 0 \quad \forall n \in N \\
& x^{l o} \leq x \leq x^{u p}
\end{array}
$$

for which a valid relaxation $(\operatorname{Rel}(F))$ is given by:

$$
\operatorname{Rel}(F)=\cap_{n \in N} \operatorname{Rel}\left(F_{n}\right)
$$

Since $\cap_{n \in N} \operatorname{Rel}\left(F_{n}\right) \subseteq \operatorname{Rel}\left(F_{n}\right), \forall n \in N$, then $\operatorname{Rel}(F) \subseteq \operatorname{Rel}\left(F_{n}\right), \forall n \in N$.

Moreover, we can establish the following Corollary. 
Corollary 2.2 If each $\operatorname{Rel}\left(F_{n}\right), n \in N$, is not dominated by any other in the set $N$, then the $\operatorname{Rel}(F)$ dominates all relaxations in the set $N$.

This corollary provides a sufficient condition for which the proposed relaxation is strictly tighter than $\operatorname{Rel}\left(F_{n}\right), n \in N$.

Remark 1: For convenience in the presentation of the formulations we have presented all the functions in every formulation as a function of the vector of variables $x$, even though different formulations $F_{n}$ may actually only involve a subset of these variables. The composite model, however, is defined fully over all variables $x$.

Remark 2: Note that since any equality constraint can be represented as the intersection of two inequalities, without loss of generality we considered the set of constraints given only by inequalities.

\section{Illustrative Example}

The following is an illustration of how different formulations may lead to different relaxed feasible regions.

Let us consider the model of a splitter shown in Figure 3. This unit is frequently used in process network models and its simple purpose is to split a main stream into substreams while keeping the same compositions. We call $f^{j}$ the inlet flow of component $j$ to the splitter and $f_{k}^{j}$ the flow of the $k-t h$ split stream of component $j$. Based on engineering knowledge the (HALR) of this problem can be described through a set of assumptions as seen in Table 1 (note that some of the information indeed may be redundant).

Table 1: High abstraction level representation for illustrative example Assumption 1 The mass of every component $j$ must be preserved as a result of the law of conservation of mass.

Assumption 2 For each outlet stream $k$ the ratio between $\frac{f_{k}^{j}}{f^{j}}$ is the same and equal to $\zeta_{k}$ for all components $j$ as defined by the splitter characteristics.

Assumption 3 The summation of the split fractions $\zeta_{k}$ for all $k$ must be one and each split fraction $\zeta_{k}$ is greater than zero and less than one.

Assumption 4 The inlet flow is bounded below and above by physical constraints. 


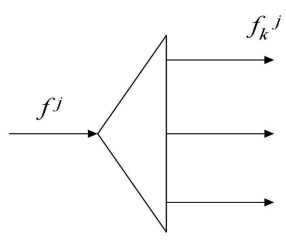

Figure 3: Splitter

Remark: Note that in this example, for illustrative purposes, we have modeled the splitter in the space of split fractions and individual flows for each component. Alternatively, as noted by Quesada and Grossmann [12], a representation in the total flow-composition space can also be developed.

The above mentioned (HALR) can be represented by at least two alternative formulations.

Formulation I:

$$
\begin{gathered}
\zeta_{k} f^{j}=f_{k}^{j} \quad \forall k, j \\
\sum_{k} f_{k}^{j}=f^{j} \quad \forall j \\
f^{j l o} \leq f^{j} \leq f^{j u p} \quad \forall j \\
0 \leq \zeta_{k} \leq 1 \quad \forall k
\end{gathered}
$$

Formulation II:

$$
\begin{gathered}
\zeta_{k} f^{j}=f_{k}^{j} \quad \forall k, j \\
\sum_{k} \zeta_{k}=1 \\
f^{j l o} \leq f^{j} \leq f^{j u p} \quad \forall j \\
0 \leq \zeta_{k} \leq 1 \quad \forall k
\end{gathered}
$$


Note that in Formulation I equation (6) is implied, and therefore, it is redundant. Similarly, in Formulation II equation (2) is implied, and therefore redundant.

The relaxation of Formulation $I$ is traditionally achieved by replacing the bilinear terms with the McCormick convex envelopes [13] :

$$
\begin{gathered}
f_{k}^{j} \leq f^{j} \zeta_{k}^{l o}+f^{j u p} \zeta_{k}-f^{j u p} \zeta_{k}^{l o} \quad \forall k, j \\
f_{k}^{j} \leq f^{j} \zeta_{k}^{u p}+f^{j l o} \zeta_{k}-f^{j l o} \zeta_{k}^{u p} \quad \forall k, j \\
f_{k}^{j} \geq f^{j} \zeta_{k}^{u p}+f^{j u p} \zeta_{k}-f^{j u p} \zeta_{k}^{u p} \quad \forall k, j \\
f_{k}^{j} \geq f^{j} \zeta_{k}^{l o}+f^{j l o} \zeta_{k}-f^{j l o} \zeta_{k}^{l o} \quad \forall k, j \\
\sum_{k} f_{k}^{j}=f^{j} \quad \forall j
\end{gathered}
$$

where $\zeta_{k}^{u p}$ and $\zeta_{k}^{l o}$ are 1 and 0 respectively. Note that this relaxation does not preserve the information in assumption 2 and assumption 3, in other words, the sum of the split fractions is not enforced to be 1 as well as the mass ratio of each component in each split.

Similarly, the relaxation of Formulation II is traditionally achieved by replacing the bilinear terms with the McCormick convex envelopes [13]:

$$
\begin{gathered}
f_{k}^{j} \leq f^{j} \zeta_{k}^{l o}+f^{j u p} \zeta_{k}-f^{j u p} \zeta_{k}^{l o} \quad \forall k, j \\
f_{k}^{j} \leq f^{j} \zeta_{k}^{u p}+f^{j l o} \zeta_{k}-f^{j l o} \zeta_{k}^{u p} \quad \forall k, j \\
f_{k}^{j} \geq f^{j} \zeta_{k}^{u p}+f^{j u p} \zeta_{k}-f^{j u p} \zeta_{k}^{u p} \quad \forall k, j \\
f_{k}^{j} \geq f^{j} \zeta_{k}^{l o}+f^{j l o} \zeta_{k}-f^{j l o} \zeta_{k}^{l o} \quad \forall k, j \\
\sum_{k} \zeta_{k}=1
\end{gathered}
$$

However, note that in this case, the relaxation does not preserve the information in assumption 1 and assumption 2, in other words, the mass balance per 
component is not enforced as well as the mass ratio in each split stream.

To summarize, we have shown that different formulations may lead to the loss of different portions of the physical meaning when they are relaxed.

In order to consider the effect on the relaxations, let us consider the case in which there are only two outlet streams $k=1,2$ for a given component $j$. Figures 4 and 5 show the projections onto sets of 2 and 3 dimensions for the feasible region defined in the relaxation of the Formulation $I$ and Formulation II, respectively. Clearly, the feasible region of both relaxations is different. For example, note that for the relaxation of Formulation II, the projection onto the split fraction $\zeta_{1,2}$ space is a segment, but a three sided polytope in the projection of the relaxation of Formulation I. Also, the projection of the relaxation of Formulation I onto the flow space is a plane, which is different from the projection of the relaxation of Formulation II onto the same space.
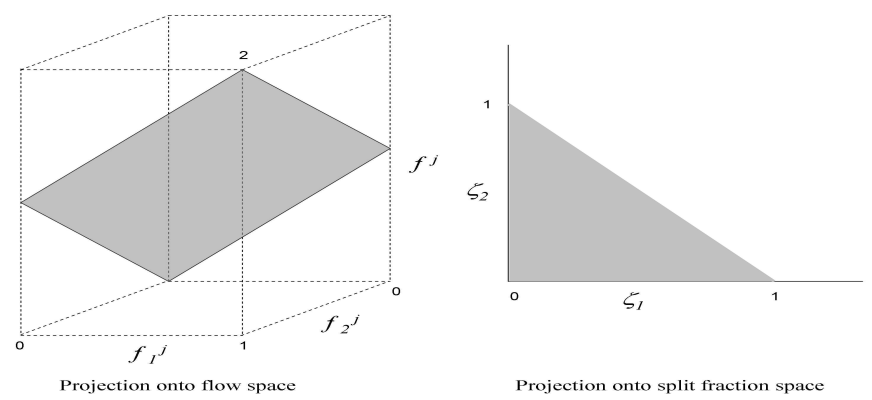

Figure 4: Projections of the relaxation for formulation I

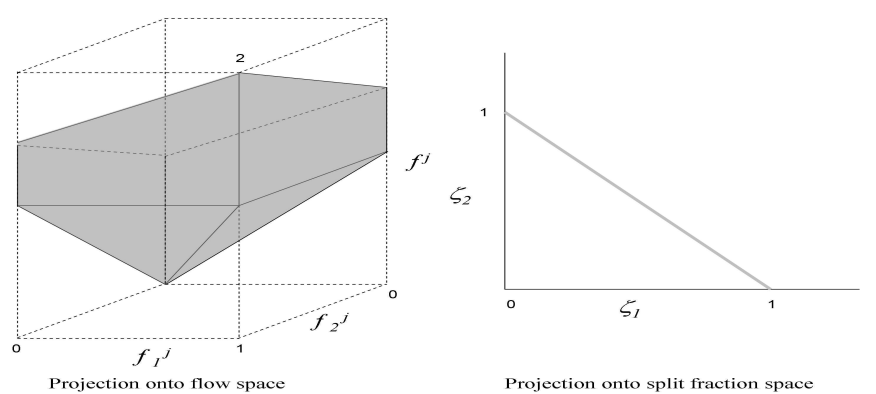

Figure 5: Projections of the relaxation for formulation II

To illustrate Theorem 2.1 we will find a relaxation for the illustrative exam- 
ple presented. The composite model reads:

$$
\begin{gathered}
\zeta_{k} f^{j}=f_{k}^{j} \quad \forall k, j \\
\sum_{k} f_{k}^{j}=f^{j} \quad \forall j \\
f^{j l o} \leq f^{j} \leq f^{j u p} \quad \forall j \\
\sum_{k} \zeta_{k}=1 \\
0 \leq \zeta_{k} \leq 1 \quad \forall k
\end{gathered}
$$

and its relaxation is:

$$
\begin{gathered}
f_{k}^{j} \leq f^{j} \zeta_{k}^{l o}+f^{j u p} \zeta_{k}-f^{j u p} \zeta_{k}^{l o} \quad \forall k, j \\
f_{k}^{j} \leq f^{j} \zeta_{k}^{u p}+f^{j l o} \zeta_{k}-f^{j l o} \zeta_{k}^{u p} \quad \forall k, j \\
f_{k}^{j} \geq f^{j} \zeta_{k}^{u p}+f^{j u p} \zeta_{k}-f^{j u p} \zeta_{k}^{u p} \quad \forall k, j \\
f_{k}^{j} \geq f^{j} \zeta_{k}^{l o}+f^{j l o} \zeta_{k}-f^{j l o} \zeta_{k}^{l o} \quad \forall k, j \\
\sum_{k} \zeta_{k}=1 \\
\sum_{k} f_{k}^{j}=f^{j} \quad \forall j
\end{gathered}
$$

As shown in Figure 6, the relaxed feasible region is tighter than the relaxation of Formulation I and Formulation II. 


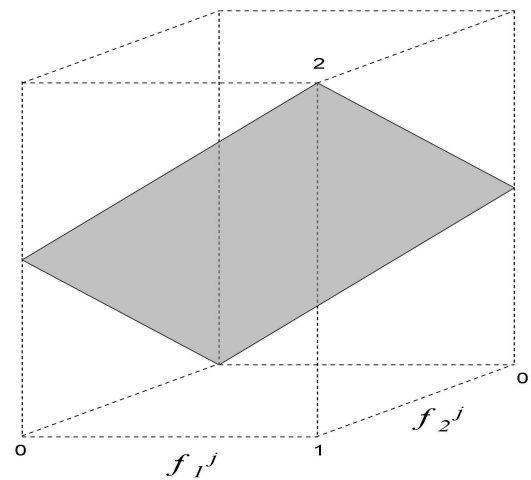

Projection onto flow space

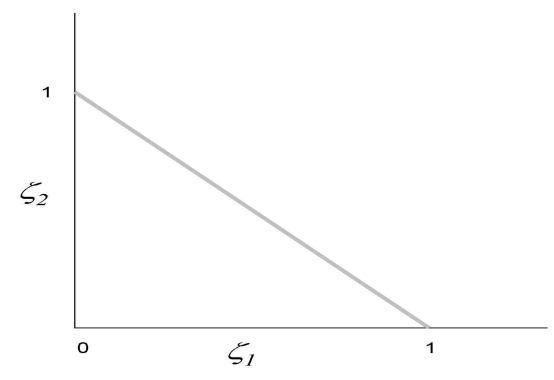

Projection onto split fraction space

Figure 6: Projections of the relaxation for composite model

\section{Implementation}

Theorem 2.1 provides a theoretical tool for generating formulations that lead to stronger relaxations. However, its direct implementation may not be of practical use for many reasons. Firstly, the $\operatorname{Rel}\left(F_{n}\right)$ may be the same for every $n \in$ $N$. Secondly, only a subset of $h_{n} \leq 0$ might be useful (i.e. their relaxations will tighten the final relaxation). Finally, adding "non-useful" constraints will increase the size of the problem without tightening the relaxation.

To overcome these potential shortcomings, we propose to consider what information is lost in the relaxation of a particular formulation and reconstruct it by using contraints from the composite model. We call this set of added constraints Generalized Reduction Constraints since they are a generalization of the work proposed by Liberti and Pantelides [11].

For example, let us consider the following formulation for a given $j \in N$ :

$$
\begin{array}{cl}
\min & Z=f(x) \\
\text { s.t. } & g(x) \leq 0 \\
& h_{j}(x) \leq 0 \\
& x^{l o} \leq x \leq x^{u p}
\end{array}
$$

where the constraints $g(x) \leq 0$ and $h_{j}(x) \leq 0$ have associated a given physical meaning described in the (HALR). Let us assume that the relaxation leads to a loss of part of that meaning. If we add a constraint $h_{i} \leq 0, h_{i}: R^{k} \rightarrow R^{p i}$, where $i \in N$ and $i \neq j$ that carries that information it will strengthen the relaxation.

This leads to the following formulation: 


$$
\begin{array}{cl}
\min & Z=f(x) \\
\text { s.t. } & g(x) \leq 0 \\
& h_{j}(x) \leq 0 \\
& h_{i}(x) \leq 0 \\
& x^{\text {lo }} \leq x \leq x^{\text {up }}
\end{array}
$$

where $h_{i}(x) \leq 0$ is called a "Generalized Reduction Constraint"

\section{$5 \quad$ Examples}

In this section we illustrate the application of the concept of Generalized Reduction Constraints in four examples.

\subsection{Process Networks}

One of the most frequent units that arises in process networks is the splitter (see Figure 3) whose main purpose is to take a stream and split it into a set of many streams as described in section 3 .

When the streams are characterized through individual flows to represent compositions we can establish the following composite model.

$$
\begin{gathered}
\zeta_{k} f^{j}=f_{k}^{j} \quad \forall k, j \\
\sum_{k} \zeta_{k}=1 \\
\sum_{k} f_{k}^{j}=f^{j} \quad \forall j
\end{gathered}
$$

A particular valid formulation for the system is given by equations (30) and (31). Since (30) are nonconvex they will need to be relaxed (e.g. by using the McCormick envelopes [13] for the bilinear terms). After this step the mass balance is not enforced anymore. Hence, adding (32) from the composite model to the formulation will strengthen the relaxation.

\subsection{Batch Reactors}

One of the optimization problems associated to batch reactors (see Figure 7) is the determination of optimal conditions for which the composition of a given component is maximized (e.g. determine the optimal time of the reaction). 


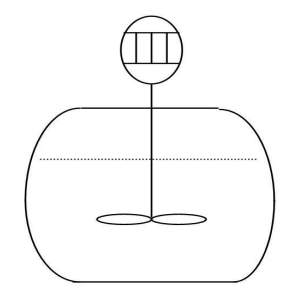

Figure 7: Batch

The relationships that often arise consist of the representation of compositions as a function of time or compositions as a function of compositions. For the particular case of two species $A$ and $B$ with exponential decay, we can establish the composite model as follows:

$$
\begin{gathered}
{[A]=e^{-k_{1} t}} \\
{[B]=e^{-k_{2} t}} \\
{[B]=[A]^{k_{2} / k_{1}}}
\end{gathered}
$$

A particular valid formulation for the system is given by equations (33) and (34). Since (33) and (34) are nonconvex they will be relaxed. In this step the relationship between $[\mathrm{A}]$ and $[\mathrm{B}]$ is lost. Hence, adding (35) from the composite model will strengthen the relaxation.

\subsection{Electric Power distribution networks (EPDN)}

One of the major assumptions in the models of Electric Power Distribution Networks is the conservation of the relationships between Active (P), Reactive (Q) and Apparent Power (S), given by the corresponding Power Phasor Diagram presented in Figure 8.

A composite model for the system can be given as:

$$
\begin{aligned}
& S \sin (\phi)=Q \\
& S \cos (\phi)=P \\
& S^{2}=P^{2}+Q^{2}
\end{aligned}
$$




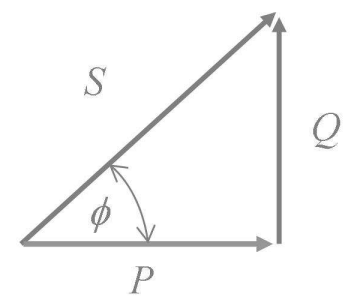

Figure 8: Power Phasor Diagram

A traditional formulation to represent this problems is given by (36) and (37). However, since they are nonconvex, they should be relaxed. After this step, the relationship between active, reactive and aparent power is lost. Adding (38) from the composite model to the formulation will strengthen the relaxation.

\subsection{Flash Units}

Separation systems that exploit the different relative volatilities of the components to be separated are often modeled by using a set of flash units (See Figure $9)$.

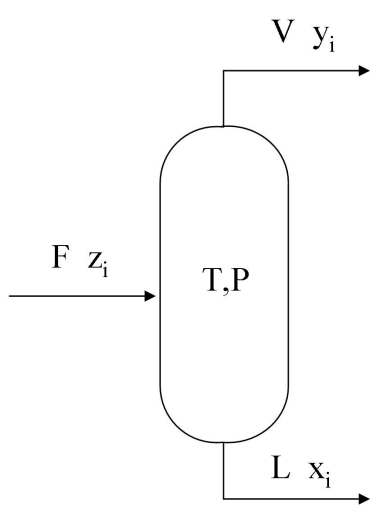

Figure 9: Flash Unit 
A composite model for a flash unit separating $i$ components from a stream of flow $F$ can be given by:

$$
\begin{gathered}
F=V+L \\
F z_{i}=V y_{i}+L x_{i} \quad \forall i \\
K_{i} x_{i}=y_{i} \quad \forall i \\
\sum_{i} x_{i}=\sum_{i} y_{i}=\sum_{i} z_{i}=1 \quad \forall i \\
F, V, L \geq 0, z_{i}, x_{i}, y_{i} \geq 0 \quad \forall i \\
\sum_{i} \frac{z_{i}\left(K_{i}-1\right)}{1+\phi\left(K_{i}-1\right)}=0
\end{gathered}
$$

where $\phi=V / F$ and $K_{i}$ the equilibrium constants. A traditional formulation to represent this problems is given by (39), (40), (41), (42) and (43). However, since some of them are nonconvex, they should be relaxed. After this step, the relationship between the compositions and flows is lost. Hence, adding (44) from the composite model to the formulation will strengthen the relaxation.

\section{$6 \quad$ Numerical Results}

In this section we present seven examples that correspond to engineering design models with the structures presented in the previous section. These examples were chosen to represent different source of nonconvexities. The parameters used for the models are given in the Appendix.

\subsection{Example 1 - Process Networks [16]}

This problem considers the selection of the optimal separation scheme to be used to separate a multicomponent process stream into a set of product streams with given purity specifications. The two components considered are A and B and they enter to the system by the feed-streams $f^{1}$ and $f^{2}$ as can be seen in Figure 10. The compositions of these streams are $55 \% \mathrm{~A}, 45 \% \mathrm{~B}$ and $50 \% \mathrm{~A}$, $50 \% \mathrm{~B}$, respectively and the desired product streams are $p^{1}$ and $p^{2}$. Purity 
specifications are a minimum of $80 \% \mathrm{~A}$ in product $p^{1}$ and a minimum of $75 \% \mathrm{~B}$ in $p^{2}$. Upper bounds are specified for the amounts of these products. Figure 10 shows a superstructure of alternative separation schemes which can be used to obtain the product. The alternatives embedded in this superstructure include: flash separation with blending, distillation with blending, flash separation and distillation in parallel, or the elimination of the complete separation process. The objective function to be maximized is the profit which is given as revenues minus costs. Revenues include the sales of $p^{1}$ and $p^{2}$, while costs include purchase costs for $f^{1}$ and $f^{2}$ as well as costs for the flash separator and distillation column which are assumed to be linear with fixed cost charges. The model $(P N)$ used for this problem can be seen below.

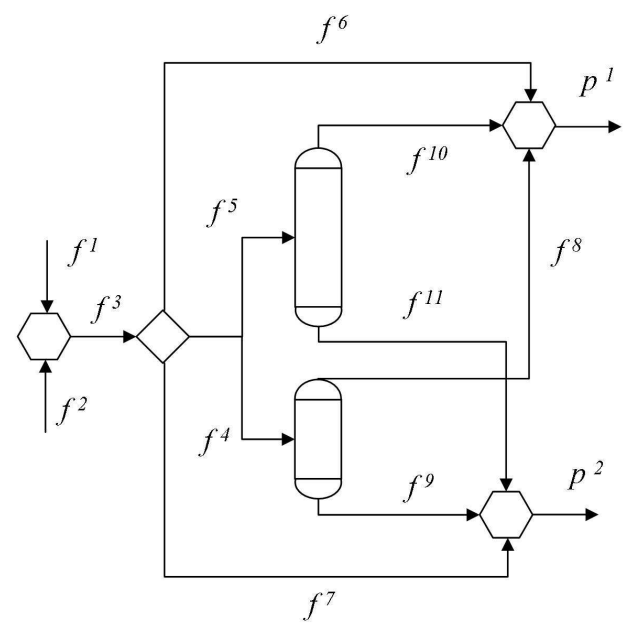

Figure 10: Process Network

$\min Z=-35 p_{a}^{1}-30 p_{b}^{2}+10 f^{1}+8 f^{2}+f_{a}^{4}+f_{b}^{4}+4 f_{a}^{5}+4 f_{b}^{5}+2 y_{f}+50 y_{d}$

$$
\begin{array}{ll}
\text { s.t. } & f_{a}^{3}=0.55 f^{1}+0.50 f^{2} \\
& p_{a}^{1}=f_{a}^{8}+f_{a}^{10}+f_{a}^{6} \\
& p_{a}^{2}=f_{a}^{9}+f_{a}^{11}+f_{a}^{7}
\end{array}
$$




$$
\begin{aligned}
& f_{b}^{3}=0.45 f^{1}+0.50 f^{2} \\
& p_{b}^{1}=f_{b}^{8}+f_{b}^{10}+f_{b}^{6} \\
& p_{b}^{2}=f_{b}^{9}+f_{b}^{11}+f_{b}^{7} \\
& f_{a}^{6}=\xi^{6} f_{a}^{3}, f_{a}^{7}=\xi^{7} f_{a}^{3} \\
& f_{a}^{4}=\xi^{4} f_{a}^{3}, f_{a}^{5}=\xi^{5} f_{a}^{3} \\
& f_{b}^{6}=\xi^{6} f_{b}^{3}, f_{b}^{7}=\xi^{7} f_{b}^{3} \\
& f_{b}^{4}=\xi^{4} f_{b}^{3}, f_{b}^{5}=\xi^{5} f_{b}^{3} \\
& \xi^{4}+\xi^{5}+\xi^{6}+\xi^{7}=1 \\
& p_{a}^{1} \geq 4.0 p_{b}^{1}, p_{a}^{1}+p_{b}^{1} \leq 15 \\
& p_{b}^{2} \geq 4.0 p_{a}^{2}, p_{a}^{2}+p_{b}^{2} \leq 18 \\
& f_{a}^{4}+f_{b}^{4} \leq 25 y_{f} \\
& f_{a}^{4}+f_{b}^{4} \geq 2.5 y_{f} \\
& f_{a}^{8}=0.85 f_{a}^{4}, f_{b}^{8}=0.20 f_{b}^{4} \\
& f_{a}^{9}=0.15 f_{a}^{4}, f_{b}^{9}=0.80 f_{b}^{4} \\
& f_{a}^{5}+f_{b}^{5} \leq 25 y_{d} \\
& f_{a}^{5}+f_{b}^{5} \geq 2.5 y_{d} \\
& f_{a}^{10}=0.975 f_{a}^{5}, f_{b}^{10}=0.050 f_{b}^{5} \\
& f_{a}^{11}=0.025 f_{a}^{5}, f_{b}^{11}=0.950 f_{b}^{5} \\
& 0 \leq f^{1}, f^{2} \leq 25 \\
& 0 \leq \xi^{4} \xi^{5} \xi^{6} \xi^{7} \leq 1, y_{f}, y_{d} \in\{0,1\}
\end{aligned}
$$

The bilinear terms that arise in the splitters lead to a nonconvex formulation. In order to solve it, a relaxation needs to be developed. In this process, the mass balance around the splitters is lost. As described in section 5.1. we propose to add the following Generalized Reduction Constraints (i.e. the mass balance per component around each splitter) to recover such information:

$$
\begin{aligned}
& f_{a}^{3}=f_{a}^{4}+f_{a}^{5}+f_{a}^{6}+f_{a}^{7} \\
& f_{b}^{3}=f_{b}^{4}+f_{b}^{5}+f_{b}^{6}+f_{b}^{7}
\end{aligned}
$$

\subsection{Example 2 - Optimal Operation of Batch Reactor}

This is a typical problem found in processes with batch reactors where reactions in series take place. In this particular case, the reactant A is converted into the component I which is subsequently converted into the component $\mathrm{P}$ following the reaction path shown below: 
Table 2: Nomenclature for Example 1

\begin{tabular}{ll}
\hline Variable & Description \\
\hline \hline$f^{i}$ & Total molar flow of stream $i, i=1,2 \ldots, 11$ \\
\hline$f_{a}^{i}$ & Total molar flow of component $a$ in stream $i, i=1,2 \ldots, 11$ \\
\hline$f_{b}^{i}$ & Total molar flow of component b in stream $i, i=1,2 \ldots, 11$ \\
\hline$\xi^{j}$ & Split fraction to stream $f^{j}, j=4,5,6,7$ \\
\hline$p_{a}^{1}$ & Molar flow of product $a$ \\
\hline$p_{b}^{2}$ & Molar flow of product $b$ \\
\hline$y_{f}$ & $0-1$ variable describing the existance/non-existance of flash unit \\
\hline$y_{d}$ & $0-1$ variable describing the existance/non-existance of distillation column \\
\hline
\end{tabular}

$$
A \stackrel{k_{1}}{\longrightarrow} I \stackrel{k_{2}}{\longrightarrow} P
$$

The following set of equations represent a differential mass balance carried out around the batch reactor considering first order kinetics for each reaction:

$$
\begin{aligned}
& \frac{d[A]}{d t}=-k_{1}[A] \\
& \frac{d[I]}{d t}=k_{1}[A]-k_{2}[I] \\
& \frac{d[P]}{d t}=k_{2}[I]
\end{aligned}
$$

The main goal of the optimization problem is to find the time necessary for the batch reactor to achieve a composition of $\mathrm{A}, \mathrm{I}$ and $\mathrm{P}$ with maximum value considering also a cost in the operating time. By integrating the set of differential equations presented above we can obtain a representation of the problem, which is explicit in time, as follows in model $(P B)$ :

$$
\begin{array}{ll}
\max & Z=p_{A}[A]+p_{I}[I]+p_{P}[P]-c_{t} t \\
& {[A]=[A]_{0} e^{-k_{1} t}} \\
& {[I]=\frac{k_{1}}{k_{2}-k_{1}}[A]_{0}\left(e^{-k_{1} t}-e^{-k_{2} t}\right)} \\
& {[P]=[A]_{0} 1-\left[\frac{k_{2}}{k_{2}-k_{1}} e^{-k_{1} t}-\frac{k_{1}}{k_{2}-k_{1}} e^{-k_{2} t}\right]} \\
& t \geq 0
\end{array}
$$

It is clear that when the exponential functions are relaxed the information that relates the concentration of the different components is lost. Hence, as described in section 5.2. we can add the following Generalized Reduction Constraints that will recover that information: 


$$
\begin{gathered}
{[I]=\frac{k_{1}}{k_{2}-k_{1}}[A]\left(\frac{[A]}{[A]_{0}}-\left(\frac{[A]}{[A]_{0}}\right)^{k_{2} / k_{1}}\right)} \\
{[P]=[A]_{0}-[I]-[A]}
\end{gathered}
$$

Table 3: Nomenclature for Example 2

\begin{tabular}{ll}
\hline Variable & Description \\
\hline \hline$[A]$ & Concentration of component A at time $t$ \\
\hline$[B]$ & Concentration of component B at time $t$ \\
\hline$[C]$ & Concentration of component C at time $t$ \\
\hline$t$ & Reaction time \\
\hline
\end{tabular}

\subsection{Example 3 - OPF Problem}

This example was taken from Debs [21] and is a particular case of an Optimal Power Flow problem which considers the minimization of the cost associated to power generation. More precisely, given two interconnected buses (see Figure 11) with predefined loads $P_{D 1}, P_{D 2}, Q_{D 1}$ and $Q_{D 2}$ we aim at finding the power to generate at each bus $P_{G 1}, P_{G 2}$ to minimize the total cost while satisfying the demand. A mathematical representation for this problem is given in model $(P O P F)$ :

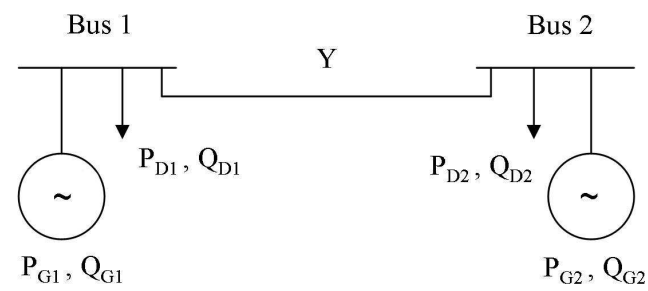

Figure 11: Electric Power Distribution Network 


$$
\begin{array}{ll}
\min & C=\left(1+P_{G 1}+3 P_{G 1}^{2}\right)+\left(2+2 P_{G 2}+P_{G 2}^{2}\right) \\
\text { s.t. } & P_{G 1}-P_{D 1}-v_{1}^{2}+v_{1} v_{2}\left(\cos \left(\delta_{2}-\delta_{1}\right)+10 \sin \left(\delta_{2}-\delta_{1}\right)=0\right. \\
& P_{G 2}-P_{D 2}-v_{2}^{2}+v_{1} v_{2}\left(\cos \left(\delta_{2}-\delta_{1}\right)-10 \sin \left(\delta_{2}-\delta_{1}\right)=0\right. \\
& Q_{G 1}-Q_{D 1}-10 v_{2}^{2}+v_{1} v_{2}\left(\sin \left(\delta_{2}-\delta_{1}\right)-10 \sin \left(\delta_{2}-\delta_{1}\right)=0\right. \\
& Q_{G 2}-P_{D 2}-v_{2}^{2}+v_{1} v_{2}\left(-\sin \left(\delta_{2}-\delta_{1}\right)-10 \cos \left(\delta_{2}-\delta_{1}\right)=0\right. \\
& P_{12}=v_{1}^{2}-v_{1} v_{2}\left(\cos \left(\delta_{2}-\delta_{1}\right)-10 \sin \left(\delta_{2}-\delta_{1}\right)\right) \\
& 0.95 \leq v_{1} \leq 1.05,0.95 \leq v_{2} \leq 1.05 \\
& 0.5 \leq P_{G 1} \leq 4,0.5 \leq P_{G 1} \leq 3.5 \\
& -0.5 \leq Q_{G 1} \leq 0.5,0.6 \leq Q_{G 1} \leq 0.6 \\
& -3 \leq P_{12} \leq 3 \\
& P_{D 1}=3, Q_{D 1}=0.3, P_{D 2}=2, Q_{D 2}=0.2
\end{array}
$$

The nonconvexities come from the trigonometric functions that arise when establishing the reactive and active power flow balances at each node. It is clear that a relaxation of these functions leads to the loss of the information that relates both powers. As described in section 5.3 the added Generalized Reduction Constraints that keep this information are:

$$
\sin \left(\delta_{i}-\delta_{j}\right)^{2}+\cos \left(\delta_{i}-\delta_{j}\right)^{2}=1 \forall i, j
$$

Table 4: Nomenclature for Example 3

\begin{tabular}{ll}
\hline Variable & Description \\
\hline \hline$P_{G i}, Q_{G i}$ & Active and Reactive power generated at Bus $i$ \\
\hline$P_{D i}, Q_{D i}$ & Active and Reactive power loads at Bus $i$ \\
\hline$v_{i}, \delta_{i}$ & Voltage and Phase at Bus $i$ \\
\hline$P_{i j}$ & Active Power in Line from Bus $i$ to Bus $j$ \\
\hline
\end{tabular}

\subsection{Example 4 - Data Reconciliation of an Isothermal Flash Unit Network}

This example corresponds to a data reconciliation problem applied to a process network with isothermal flash units. The optimization, control or operation of 
a process often relies on having measurements (e.g. flows, compositions) that can be used to adjust models. In general, the number of sensors in a plant is larger than the degrees of freedom of the models. However, the information they provide is often not accurate due to noise of measurements or failures in the sensors. In order to infer better values for the measurements, a data reconciliation is necessary previous to their use for optimization or control. As shown in Figure 12 the process network we are aiming at reconciling consists of a set of three flash units separating a stream with three components, propane, butane and pentane. The goal is to minimize the sum of the square of the differences between the measured values and the values calculated by the model. A mathematical representation of the problem can be found in model $(P D R)$.

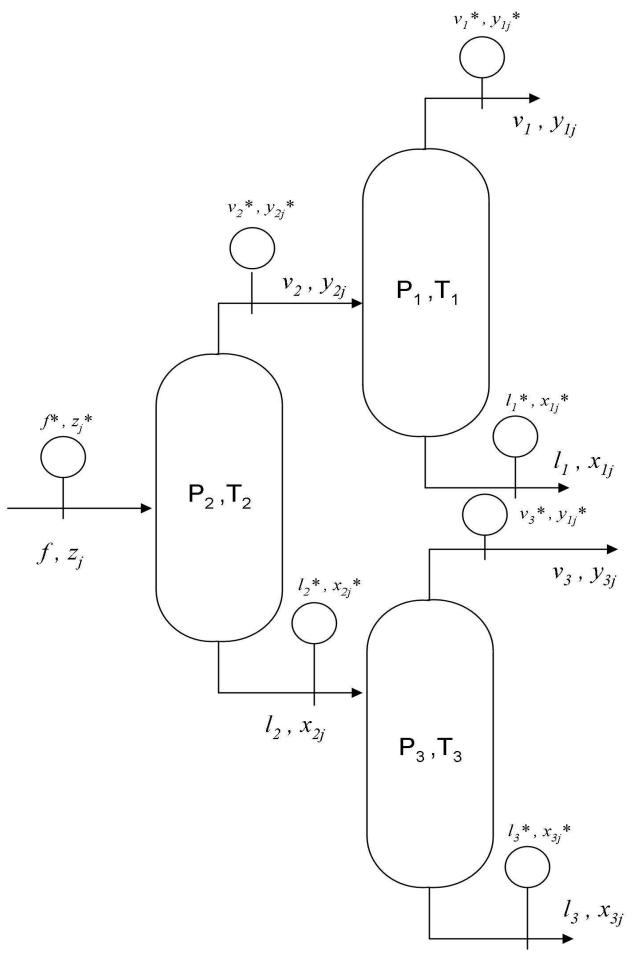

Figure 12: Flash Unit Network 


$$
\begin{array}{ll}
\min & Z=\sum_{i} \sum_{j}\left(\left(x_{i j}^{*}-x_{i j}\right)^{2}+\left(y_{i j}^{*}-x_{i j}\right)^{2}\right)+\left(z_{i j}^{*}-z_{i j}\right)^{2}+ \\
& \sum_{i}\left(\left(v_{i}^{*}-v_{i}\right)^{2}+\left(l_{i}^{*}-l_{i}\right)^{2}\right)+\left(f^{*}-f\right)^{2} \\
\text { s.t. } & f=v_{2}+l_{2} \\
& f_{2} z_{j}=v_{2} y_{2 j}+l_{2} x_{2 j} \quad \forall j \\
& v_{2}=v_{1}+l_{1} \\
& v_{2} y_{2 j}=v_{1} y_{1 j}+l_{1} x_{1 j} \quad \forall j \\
& l_{2}=v_{3}+l_{3} \\
& l_{2} x_{2 j}=v_{3} y_{3 j}+l_{3} x_{3 j} \quad \forall j \\
& \sum_{j} x_{i j}=\sum_{j} y_{i j}=\sum_{j} z_{j}=1 \quad \forall i \\
& K_{i j} x_{i j}=y_{i j} \quad \forall i, j \\
& f, v_{i}, l_{i} \geq 0 \quad \forall i \\
& x_{i j}, y_{i j}, z_{j} \geq 0 \quad \forall i, j
\end{array}
$$

As described in section 5.4 a Generalized Reduction Constraint suitable for this system comes from the Rachford-Rice equation [20] which keeps the relationship between the vaporized fraction and the inlet compositions, as shown below:

$$
\begin{aligned}
& \sum_{j} \frac{z_{j}\left(K_{2 j}-1\right)}{1+\phi_{2}\left(K_{2 j}-1\right)}=0 \\
& \sum_{j} \frac{y_{2 j}\left(K_{1 j}-1\right)}{1+\phi_{1}\left(K_{1 j}-1\right)}=0 \\
& \sum_{j} \frac{x_{2 j}\left(K_{3 j}-1\right)}{1+\phi_{3}\left(K_{3 j}-1\right)}=0
\end{aligned}
$$

where $\phi_{1}=v_{1} / v_{2}, \phi_{2}=v_{2} / f$ and $\phi_{3}=v_{3} / l_{2}$

Table 5: Nomenclature for Example 4

\begin{tabular}{ll}
\hline Variable & Description \\
\hline \hline$f, z_{j}$ & Feed molar flow and molar fraction of component $j$ to flash tank 2 \\
\hline$v_{i}, x_{i j}$ & Vapor flow and molar fraction of component $j$ from flash tank $i$ \\
\hline$l_{i}, y_{i j}$ & Liquid flow and molar fraction of component $j$ from flash tank $i$ \\
\hline
\end{tabular}




\subsection{Example 5 - Water Treatment Networks}

In the Water Treatment Network Design Problem [22], we are given a set of contaminated water streams for which the composition of contaminants and molar flows are perfectly defined. The goal of this problem is to find a set of treatment units with known treating performances and interconnections which will minimize the cost of the network while satisfying maximum concentrations of contaminants in the outlet stream. In this particular case study we only consider two contaminants. A general scheme of the system is shown in Figure 13. A mathematical representation for the problem is given by model $(P T R)$.

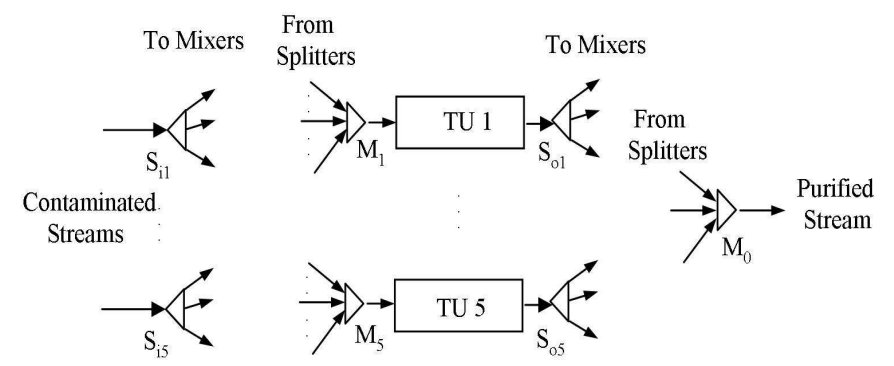

Figure 13: Water Treatment Superstructure for Example 5

$$
\begin{array}{cl}
\min & z=\sum_{k} C T U_{k} \\
\text { s.t. } & \\
& f_{i j k}=\xi_{i}^{k} f_{i j} \quad i \in I, j \in J, k \in K \\
& f_{i j}^{0}=\xi_{i}^{0} f_{i j} \quad i \in I, j \in J \\
& \xi_{i}^{0}+\sum_{k} \xi_{i}^{k}=1 \quad i \in I \\
& f_{k j k^{\prime}}=\xi_{k}^{k^{\prime}} f o_{k j} \quad k^{\prime}, k \in K, j \in J \\
& f_{k j}^{0}=\xi_{k}^{0} f o_{k j} \quad j \in J, k \in K \\
& \xi_{k}^{0}+\sum_{k^{\prime}} \xi_{k}^{k^{\prime}}=1 \quad j \in J, k \in K \\
& f_{k j}=\left(1-C_{k j}\right) f i_{k j} \quad j \in J k \in K \\
& \sum_{k} f_{k j}^{0}+\sum_{i} f_{i j}^{0} \leq T_{j} \quad j \in J \\
& \sum_{j} f i_{k j} \geq L_{k} Y_{k} \quad k \in K \\
& C T U_{k}=\beta_{k} \sum_{j} f i_{k j}+\gamma_{k} Y_{k}+\theta_{k}\left(\sum_{j} f i_{k j}\right) \\
& \sum_{j} f i_{k j} \leq \sum_{j} f i_{k j}^{u p} Y_{k} \\
& f_{i j k} \geq 0, i \in I, j \in J, k \in K \\
& \xi_{k}^{k^{\prime}} \geq 0 \quad k, k^{\prime} \in K \\
& \xi_{i}^{k} \geq 0 \quad i \in I, k \in K
\end{array}
$$




$$
\begin{aligned}
& f_{k j k^{\prime}} \geq 0 \quad k, k^{\prime} \in K, j \in J \\
& Y_{k} \in\{0,1\} \quad k \in K
\end{aligned}
$$

As in Example 1 the bilinear terms that arise in the splitters lead to a nonconvex formulation. In order to solve it a relaxation needs to be developed. In this process, the mass balance around the splitters is lost. As described in section 5.1, we propose to add the following Generalized Reduction Constraints, which corresponds to mass balances for each component around each splitter from treatment unit $\mathrm{K}$, to recover such information.

$$
\sum_{k^{\prime} \in K} f_{k j k^{\prime}}+f_{k j}^{0}=f o_{k j} \quad j \in J, k \in K
$$

It is important to note that surrogates of these constraints can be added to reduce the increase in the size of the formulation as we will present in Example 6 and 7 .

Table 6: Nomenclature for Example 5

\begin{tabular}{ll}
\hline Variable & Description \\
\hline \hline$C T U_{k}$ & Cost of treatment unit $k$ \\
\hline$f_{i j k}$ & Molar flow of component $j$ from splitter $i$ going to mixer $k$ \\
\hline$f_{k j k^{\prime}}$ & Molar flow of component $j$ from splitter $k$ going to mixer $k^{\prime}$ \\
\hline$\xi_{i}^{k}$ & Split fraction of splitter i going to mixer k \\
\hline$\xi_{k^{\prime}}^{k}$ & Split fraction of splitter $k^{\prime}$ going to outlet stream mixer \\
\hline$\xi_{i}^{o}$ & Split fraction of splitter $i$ going to outlet stream mixer \\
\hline$\xi_{k^{\prime}}^{o}$ & Split fraction of splitter $k^{\prime}$ going to mixer $k$ \\
\hline$f o_{k j}$ & Molar flow of component $j$ leaving treatment unit $k$ \\
\hline$f i_{k j}$ & Molar flow of component $j$ entering treatment unit $k$ \\
\hline$f i_{k j}^{0}$ & Molar flow of component $j$ from splitter $k$ leaving system \\
\hline$y_{k}$ & 0-1 variable describing the selection/no-selection of treatment unit k \\
\hline$S e t s$ & Description \\
\hline \hline$I$ & Set of all the splitters receiving contaminated water from the process \\
\hline$K$ & Set of treatment units \\
\hline$J$ & Set of components of the streams \\
\hline
\end{tabular}




\subsection{Example 6 and Example 7 - Integrated Process Net- works}

Given is a set of process units that generate contaminants and a set of treatment units that selectively remove these contaminants. The problem is to find an integrated network of the process units with the water treatment units interconnected by mixers and splitters in order to minimize the freshwater and wastewater flows, or more generally the total cost of constructing and operating the network. In order to address this problem, a superstructure is developed as shown in Figures 14 and 15. Here, a freshwater source is present at the inlet of the network, from which freshwater is fed to the process units. These process units, in turn, are interconnected in all possible ways and also to the treatment units by making use of mixers and splitters. Similarly, the treatment units, which are treated simply with fixed recoveries, are also interconnected in all possible ways and with a final single stream that is discharged into the environment. There is also an option of bypassing wastewater generated in the process units directly to the discharge without any treatment. For this synthesis problem, the water flow demands of the process units are assumed to be fixed. As described in Karuppiah and Grossmann [17], a formulation for this system is given by model $(P W N)$.

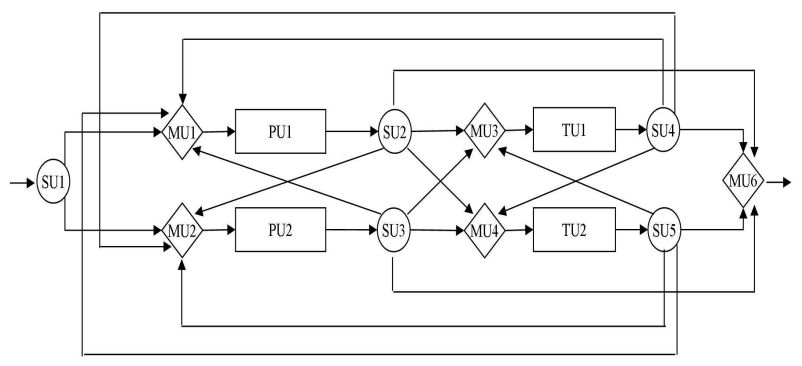

Figure 14: Water Treatment Superstructure for example 6 


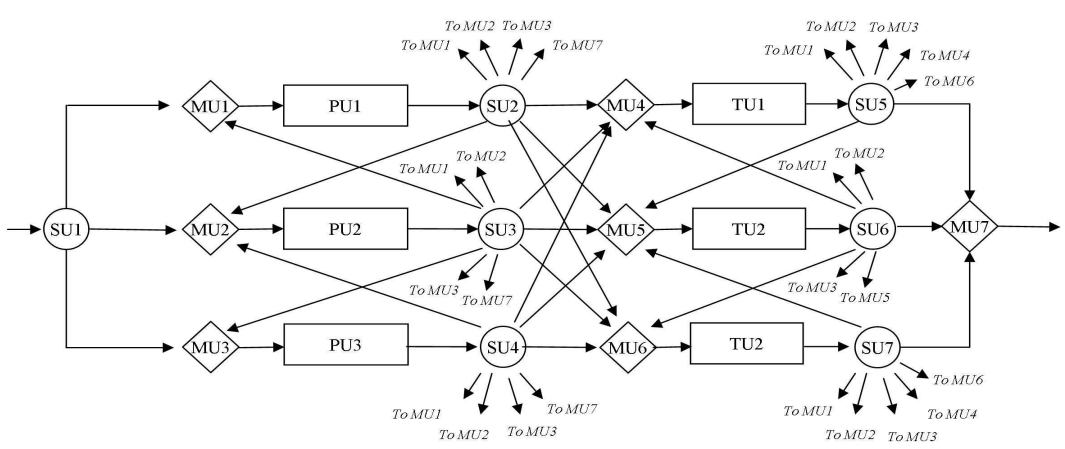

Figure 15: Water Treatment Superstructure for example 7

$$
\begin{array}{ll}
\min \quad & Z=H C_{F C} F W+A R \sum_{t \in T U} I N V^{t}+A R \sum_{d \in C N} P I N V^{d}+ \\
& H \sum_{t \in T U} O P^{t}+H \sum_{d \in C N} P O P^{d} \\
\text { s.t. } \quad & F^{k}=\sum_{i \in m_{\text {in }}} F^{i} \quad \forall m \in M U, \forall k \in m_{\text {out }} \\
& F^{k} C_{j}^{k}=\sum_{i \in m_{\text {in }}} F^{i} C_{j}^{i} \quad \forall j, \forall m \in M U, \forall k \in m_{\text {out }} \\
& F^{k}=\sum_{i \in s_{\text {out }}} F^{i} \quad \forall s \in S U, \forall k \in s_{\text {in }} \\
& C_{j}^{i}=C_{j}^{k} \quad \forall j, \forall s \in S U, \forall i \in s_{\text {out }}, \forall k \in s_{\text {in }} \\
& F^{k}=F^{i}=P^{p} \quad \forall p \in P U, \forall i \in p_{\text {in }}, \forall k \in p_{\text {out }} \\
& P^{p} C_{j}^{i}+L_{j}^{p} \times 10^{3}=P^{p} C_{j}^{k} \quad \forall j, \forall p \in P U, \forall i \in p_{\text {in }}, \forall k \in p_{\text {out }}, \\
& F^{k}=F^{i} \quad \forall t \in T U, \forall i \in t_{\text {out }}, \forall k \in t_{\text {in }} \\
& C_{j}^{i}=\beta_{j}^{t} C_{j}^{k} \quad \forall j, \forall t \in T U, \forall i \in t_{\text {out }}, \forall k \in t_{\text {in }} \\
& T I N V^{t}=\gamma^{t}\left(F^{i}\right)^{\alpha} \quad \forall t \in T U, \forall i \in t_{\text {out }} \\
& T O P^{t}=\theta^{t} F^{i} \quad \forall t \in T U, \forall i \in t_{\text {out }} \\
& P I N V^{d}=\omega_{\text {pip }}\left(F^{d}\right)^{\alpha}+\omega_{\text {inst }} y p_{d} \\
& P O P^{d}=\omega_{\text {pum }} F^{d} \quad \forall d \in C N \\
& F^{d L} y p_{d} \leq F^{d} \leq F^{d U} y p_{d} \forall d \in C N \\
& C^{L} \leq C \leq C^{U} \quad \\
& F^{L} \leq F \leq F^{U} \quad \\
& y p_{d} \in\{0,1\} \forall d \in C N \quad \\
& y t_{t} \in\{0,1\} \forall t \in T U
\end{array}
$$

As in Examples 1 and 5, the non-convexities lead to a violation of the mass balance in the relaxation. A Generalized Reduction Constraint that recovers this information for the global mass balance is as follows: 


$$
\sum_{p \in P U} L_{j}^{p} \times 10^{3}=\sum_{\substack{t \in T U \\ i \in t_{i n}}}\left(1-\beta_{j}^{t} F^{i} C_{j}^{i}\right)+F^{\text {out }} C_{j}^{\text {out }} \forall j
$$

Note that this constraint can be thought as a surrogate of all the constraints that would arise if we enforced the mass balance around each unit operation.

Table 7: Nomenclature for Example 6 and 7

\begin{tabular}{ll}
\hline Variable & Description \\
\hline \hline$C_{j}^{i}$ & Concentration of contaminant $j$ in stream $i$ \\
\hline$f_{j}^{i}$ & Flow of contaminant $j$ in stream $i$ \\
\hline$f_{j}^{\text {out }}$ & Flow of contaminant $j$ in the outlet stream to the environmnet \\
\hline$F^{i}$ & Flowrate of stream $i$ \\
\hline$F W$ & Fresh water intake into the system \\
\hline$T I N V^{t}$ & Investment cost for treatment unit $t$ \\
\hline$T O P^{t}$ & Operating cost for treatment unit $t$ \\
\hline$P I N V^{d}$ & Investment cost for pipe and pump $d$ \\
\hline$P O P^{d}$ & Operation cost for pump and pipe $d$ \\
\hline
\end{tabular}

Table 8: Nomenclature for Example 6 and 7

\begin{tabular}{ll}
\hline Sets & Description \\
\hline \hline$m_{\text {in }}$ & Set of inlet streams into mixer $m$ \\
\hline$m_{\text {out }}$ & Outlet stream from mixer $m$ \\
\hline$M U$ & Set of mixers \\
\hline$p_{\text {in }}$ & Inlet stream into process unit $p$ \\
\hline$p_{\text {out }}$ & Outlet stream from process unit $p$ \\
\hline$P U$ & Set of process units \\
\hline$s_{\text {in }}$ & Inlet stream into splitter $s$ \\
\hline$s_{\text {out }}$ & Outlet streams from splitter $s$ \\
\hline$S U$ & Set of splitters $s$ \\
\hline$t_{\text {in }}$ & Inlet stream into treatment unit $t$ \\
\hline$t_{\text {out }}$ & Outlet streams from treatment unit $t$ \\
\hline$T U$ & Set of treatment units \\
\hline$C N$ & Set of all connections between units \\
\hline
\end{tabular}




\subsection{Computational Results}

In order to assess the performance of the method we solve the problems using the commercial global optimization solver BARON [15] version 9.0.3 and analyse the lower bounds obtained at the root node, number of nodes generated in the search process and computational times. This analysis is carried out in both, the formulation with and without Generalized Reduction Constraints. Note that Example 3 requires a special treatment since BARON is not able to handle trigonometric functions. In this case we use a customized implementation of a spatial branch and bound algorithm which uses BARON in a lower level, to solve the bilinear and trilinear problems resulting from the relaxation of the trigonometric functions. All problems were solved using a Pentium(R) CPU $3.40 \mathrm{GHz}$ and $1 \mathrm{~GB}$ of RAM.

Table 9: Size and Characteristics of Examples

\begin{tabular}{|c|c|c|c|c|}
\hline Example & $\begin{array}{c}\text { Integer } \\
\text { Variables }\end{array}$ & $\begin{array}{c}\text { Continuous } \\
\text { Variables }\end{array}$ & Constraints & $\begin{array}{c}\text { Generalized Reduction } \\
\text { Constraints }\end{array}$ \\
\hline \hline 1 & 2 & 31 & 22 & 2 \\
\hline 2 & 0 & 4 & 4 & 2 \\
\hline 3 & 0 & 8 & 6 & 1 \\
\hline 4 & 0 & 29 & 31 & 25 \\
\hline 5 & 5 & 421 & 380 & 2 \\
\hline 6 & 24 & 123 & 135 & 2 \\
\hline 7 & 53 & 227 & 245 & 3 \\
\hline
\end{tabular}

The size and main features of the 7 test problems is shown in Table 9. As shown in Table 10, examples 1,2, 3 and 4 show a significant improvement in the lower bound predicted at the root node as well as a significant decrease in the number of nodes when using the proposed approach. Since these examples are small in size (see Table 9), the tighter relaxation has little effect in the computational times. Examples 5, 6 and 7, which are larger in size, clearly show the benefits of using the proposed formulations by significantly decreasing the computational requirements. Overall, more than $90 \%$ reduction in the number of nodes visited to find the solution and more than $80 \%$ improvement in the gap between the lower bound and upper bound at the root node is a clear indication that the formulations proposed lead to tighter relaxations. It is also important 
Table 10: Performance of Spatial Branch and Bound using different formulation strategies*

\begin{tabular}{|c|c|c|c|c|c|c|c|}
\cline { 3 - 8 } \multicolumn{2}{c|}{} & \multicolumn{3}{c|}{ Traditional Approach } & \multicolumn{3}{c|}{ Proposed Approach } \\
\hline Instance & GO & LB & Nodes & Time $(\mathrm{s})$ & LB & Nodes & Time(s) \\
\hline 1 & -510.08 & -792.2 & 37 & $<1$ & -510.08 & 1 & $<1$ \\
\hline 2 & -1.49 & -1.53 & 17 & $<1$ & -1.49 & 1 & $<1$ \\
\hline 3 & 24.05 & 23.5 & 20 & 30 & 24.05 & 1 & 1 \\
\hline 4 & 22.58 & 21.98 & 91 & 4 & 22.58 & 1 & $<1$ \\
\hline 5 & 348,337 & 291,573 & 3,588 & 174 & 293,318 & 1,444 & 74 \\
\hline 6 & 253,676 & 32,486 & 40,000 & $>3,600$ & 250,961 & 138 & 9 \\
\hline 7 & $1,347,456$ & 987,139 & 33,800 & $>3,600$ & $1,208,590$ & 3,563 & 209 \\
\hline
\end{tabular}

*All examples were solved fully using BARON except for example 3 which used a

customized global optimization algorithm

to note that, in Examples 6 and 7, the lack of Generalized Reduction Constraints leads to formulations that are unsolvable within a 1 hour limit.

\section{Conclusions}

In this work we have presented a methodology to generate formulations for nonconvex MINLPs that lead to stronger relaxations. The novelty of this approach is based on the consideration that relaxations lead to a loss in the information necessary to describe the high abstraction level representation (HALR). The theoretical results for tighter relaxations were supported by a set of seven examples found in different areas such as process networks, batch reactors, electric power networks, data reconciliation and water networks. Large reduction in number of nodes and computational times were verified when applying global optimization solvers to three large scale problems (Examples 5-7). 


\section{References}

[1] Grossmann, I.E. And Kravanja Z., Mixed-integer nonlinear programming: a survey of algorithms and applications. In: Large-scale optimization with applications, part II: optimal design and control, Berlin:Springer, 1997.

[2] Benders J. F. , Partitioning procedures for solving mixed-variables programming problems. Numerische Mathematik,4, 238-252, 1962.

[3] Geoffrion A. M., Generalized Benders decomposition., JOTA 10, 237$260,1972$.

[4] Duran, M. A. and Grossmann, I. E.,An outer-approximation algorithm for a class of mixed-integer nonlinear programs., Mathematical Programming, 36, 307, 1986.

[5] Tawarmalani, M. and Sahinidis, N., Convexification and Global Optimization in Continuous and Mixed-Integer Nonlinear Programming., Kluwer Academic Publishers, 2002.

[6] Horst, R. And Tuy, H., Global Optimization deterministic approaches (3rd Ed), Berlin: Springer-Verlag, 1996.

[7] Floudas C.A., Deterministic Global Optimization: Theory, Methods and Applications, Kluwer Academic Publishers, Dordrecht, The Netherlands, 2000.

[8] Falk J.E. And Soland R.M., An Algorithm for Separable Nonconvex Programming Problems, Management Science, 15:9, 550-569, 1969.

[9] Sherali, H. D. and Alameddine,A.,A new reformulation linearization technique for bilinear programming problems, Journal of Global Optimization 2, 379-410, 1992.

[10] Liberti L. Reduction Constraints for the Global Optimization of NLPs., International Transactions in Operation Research, 11:1, 33-41,2004.

[11] Liberti, L. and Pantelides, C. C. An exact reformulation algorithm for large nonconvex NLPs involving bilinear terms., Journal of Global Optimization, 36:2, 161-189,2006. 
[12] Quesada, I. and Grossmann I. E., Global optimization of bilinear process networks with multicomponent flows, Computers and Chemical Engineering, 19(12), 1219-1242, 1995b.

[13] McCormick, G. P., Computability of global solutions to factorable nonconvex programs. Part I. Convex underestimating problems., Mathematical Programming, 10, 146-175, 1976.

[14] Westerlund, T. and Pettersson, F., An extended cutting plane method for solving convex MINLP problems., Computers Chem. Eng. Sup. 19:131175, 1995.

[15] SAHINidis, N. V., BARON: A general purpose global optimization software package, Journal of Global Optimization, 8:2, 201-205, 1996.

[16] Kocis G.R. and Grossmann I.E., Relaxation Strategy for the structural optimization of Process Flow Sheets, Industrial and Engineering Chemistry Research, 26,1869, 1987.

[17] Karuppiah R. and Grossmann I. E., Global optimization for the synthesis of integrated water systems in chemical processes, Computers and Chemical Engineering,30:4,650-673,2006.

[18] Lindo Systems Inc, LindoGLOBAL Solver

[19] Adjiman C.S., Dallwig S., Floudas C.A. and Neumaier A., A Global Optimization Method, $\alpha B B$, for General Twice-Differentiable NLPs - I. Theoretical Advances, Computers and Chemical Engineering, 22, 1137, 1998.

[20] Whitson C., Michelsen M.L., The Negative Flash, Fluid Phase Equilibria, 53,5171, 1989.

[21] Debs A.S.,Load Flow Analysis, Power Systems Analysis and Planning Proceedings of the Arab School on Science and Technology, 1983.

[22] Ruiz, J.P. and Grossmann I.E.,Water Treatment Network Design, Minlp Cyber-Infrastructure - www.minl.org, 2009. 


\section{Appendix}

Table 11: Parameters for Example 2

\begin{tabular}{|c|c|c|}
\hline Parameter & Description & Value \\
\hline \hline$[A]_{0}$ & Initial concentration of A & 1 \\
\hline$k_{1}$ & Kinetic constant for first reaction & 0.05 \\
\hline$k_{2}$ & Kinetic constant for second reaction & 0.05 \\
\hline$p_{a}$ & Price of component A & 1 \\
\hline$p_{b}$ & Price of component B & 3 \\
\hline$p_{c}$ & Price of component C & 4 \\
\hline$c_{t}$ & Penalty on the processing time & 0.05 \\
\hline
\end{tabular}

Table 12: Values for partition coefficients $K_{i j}$ for example 4

\begin{tabular}{lccc}
\hline$i / j$ & Propane & Butane & Pentane \\
\hline \hline 1 & 2.184 & 1.665 & 0.187 \\
\hline 2 & 1.927 & 0.514 & 0.155 \\
\hline 3 & 1.692 & 0.438 & 0.128 \\
\hline
\end{tabular}

Table 13: Measurements for molar fraction composition $x_{i j}^{*}$ for example 4

\begin{tabular}{cccc}
\hline$i / j$ & Propane & Butane & Pentane \\
\hline \hline 1 & 0.2 & 0.3 & 0.5 \\
\hline 2 & 0.2 & 0.6 & 0.1 \\
\hline 3 & 0.1 & 0.3 & 0.4 \\
\hline
\end{tabular}


Table 14: Measurements for molar fraction composition $y_{i j}^{*}$ for example 4

\begin{tabular}{cccc}
\hline$i / j$ & Propane & Butane & Pentane \\
\hline \hline 1 & 0.2 & 0.5 & 0.8 \\
\hline 2 & 0.2 & 0.5 & 0.8 \\
\hline 3 & 0.2 & 0.5 & 0.8 \\
\hline
\end{tabular}

Table 15: Measurements for molar fraction composition $z_{j}^{*}$ for example 4

\begin{tabular}{lccc}
\hline $\mathrm{i} / \mathrm{j}$ & Propane & Butane & Pentane \\
\hline \hline Flash Tank 2 & 0.2 & 0.5 & 0.3 \\
\hline
\end{tabular}

Table 16: Measurements for molar flows $v^{*}, l^{*}$ and $f^{*}$ for example 4

\begin{tabular}{lccc}
\hline stream/tank & 1 & 2 & 3 \\
\hline \hline $\mathrm{v}^{*}$ & 7 & 18 & 20 \\
\hline $\mathrm{l}^{*}$ & 10 & 16 & 5 \\
\hline $\mathrm{f}^{*}$ & - & 36 & - \\
\hline
\end{tabular}

Table 17: Inlet flow of the component $j$ in the splitter $i\left(f_{i j}\right)$ for example 5

\begin{tabular}{lccccc}
\hline TU/Component & 1 & 2 & 3 & 4 & 5 \\
\hline \hline TU 1 & 1.0 & 1.0 & 1.0 & 1.0 & 10.0 \\
\hline TU 2 & 2.0 & 0.0 & 0.0 & 1.0 & 15.0 \\
\hline TU 3 & 1.0 & 0.0 & 1.0 & 2.0 & 5.0 \\
\hline TU 4 & 3.0 & 1.0 & 1.0 & 0.0 & 20.0 \\
\hline TU 5 & 2.0 & 2.0 & 0.0 & 0.0 & 10.0
\end{tabular}


Table 18: Performance coefficient for each component and unit $\left(C_{k j}\right)$ for example 5

\begin{tabular}{lcccc}
\hline TU/Component & 1 & 2 & 3 & 4 \\
\hline \hline TU 1 & 0.90 & 0.70 & 0.60 & 0.00 \\
\hline TU 2 & 0.85 & 0.90 & 0.60 & 0.00 \\
\hline TU 3 & 0.00 & 0.60 & 0.85 & 0.95 \\
\hline TU 4 & 0.00 & 0.60 & 0.60 & 0.85 \\
\hline TU 5 & 0.00 & 0.60 & 0.85 & 0.30 \\
\hline
\end{tabular}

Table 19: Minimum flow limit for equipment $\left(L_{k}\right)$ for example 5

\begin{tabular}{|c|c|c|c|c|}
\hline TU 1 & TU 2 & TU 3 & $\mathrm{TU}$ & TU 5 \\
\hline 5.0 & 3.0 & 4.0 & 3.0 & 1.0 \\
\hline
\end{tabular}

Table 20: Maximum contaminant flow limit in the outlet $\left(L_{k}\right)$ for example 5

\begin{tabular}{cccc}
\hline 1 & 2 & 3 & 4 \\
\hline \hline 3.0 & 3.0 & 3.0 & 3.0 \\
\hline
\end{tabular}

Table 21: Cost coefficients for unit $k$ for example 5

\begin{tabular}{lccccc}
\hline Coefficient & TU 1 & TU 2 & TU 3 & TU 4 & TU 5 \\
\hline \hline$\theta_{k}$ & 1000.0 & 1300.0 & 3000.0 & 2000.0 & 1000.0 \\
\hline$\beta_{k}$ & 6000.0 & 6000.0 & 6000.0 & 6000.0 & 6000.0 \\
\hline$\gamma_{k}$ & 0.0 & 0.0 & 0.0 & 0.0 & 0.0 \\
\hline
\end{tabular}

Table 22: Process Unit Data for Example 6

\begin{tabular}{lllllll}
\hline Unit & $\begin{array}{l}\text { Flowrate } \\
(\mathrm{t} / \mathrm{h})\end{array}$ & $\begin{array}{l}\text { Discharge } \\
\text { Load }(\mathrm{kg} / \mathrm{h})\end{array}$ & \multicolumn{2}{l}{$\begin{array}{l}\text { Max Inlet } \\
\text { Conc. (ppm) }\end{array}$} & $\alpha$ \\
\cline { 3 - 6 } & & $\mathrm{A}$ & $\mathrm{B}$ & $\mathrm{A}$ & $\mathrm{B}$ & \\
\hline PU1 & 40 & 1 & 1.5 & 0 & 0 & 0.7 \\
\hline PU2 & 50 & 1 & 1 & 50 & 50 & 0.7 \\
\hline
\end{tabular}


Table 23: Treatment Unit Data for Example 6

\begin{tabular}{|c|c|c|c|c|}
\hline \multirow[t]{2}{*}{ Unit } & \multicolumn{2}{|c|}{$\begin{array}{l}\text { Removal } \\
\text { Ratio (\%) }\end{array}$} & \multirow[t]{2}{*}{$\begin{array}{l}\text { Investment } \\
\text { Cost }(\gamma)\end{array}$} & \multirow{2}{*}{$\begin{array}{l}\text { Operating } \\
\text { Cost }(\theta) \\
\theta\end{array}$} \\
\hline & $\mathrm{A}$ & B & & \\
\hline TU1 & 95 & 0 & 16800 & 1 \\
\hline TU2 & 0 & 95 & 12600 & 0.0067 \\
\hline
\end{tabular}

Table 24: Process Unit Data for Example 7

\begin{tabular}{lllllll}
\hline Unit & $\begin{array}{l}\text { Flowrate } \\
(\mathrm{t} / \mathrm{h})\end{array}$ & \multicolumn{2}{l}{$\begin{array}{l}\text { Discharge } \\
\text { Load }(\mathrm{kg} / \mathrm{h})\end{array}$} & \multicolumn{2}{l}{$\begin{array}{l}\text { Max Inlet } \\
\text { Conc. (ppm) }\end{array}$} & \multirow{2}{*}{$\alpha$} \\
\cline { 3 - 6 } & & $\mathrm{A}$ & $\mathrm{B}$ & $\mathrm{A}$ & $\mathrm{B}$ & \\
\hline PU1 & 40 & 1 & 1.5 & 0 & 0 & 0.7 \\
\hline PU2 & 50 & 1 & 1 & 50 & 50 & 0.7 \\
\hline PU3 & 60 & 1 & 1 & 50 & 50 & 0.7 \\
\hline
\end{tabular}

Table 25: Treatment Unit Data for Example 7

\begin{tabular}{|c|c|c|c|c|}
\hline \multirow[t]{2}{*}{ Unit } & \multicolumn{2}{|c|}{$\begin{array}{l}\text { Removal } \\
\text { Ratio (\%) }\end{array}$} & \multirow[t]{2}{*}{$\begin{array}{l}\text { Investment } \\
\text { Cost }(\gamma)\end{array}$} & \multirow[t]{2}{*}{$\begin{array}{l}\text { Operating } \\
\text { Cost }(\theta)\end{array}$} \\
\hline & $\mathrm{A}$ & B & & \\
\hline TU1 & 95 & 0 & 16800 & 1 \\
\hline TU2 & 80 & 90 & 24000 & 0.033 \\
\hline TU3 & 0 & 95 & 12600 & 0.0067 \\
\hline
\end{tabular}

Table 26: Pump and Piping Data for Example 6-7

\begin{tabular}{lll}
$\omega_{\text {pip }}$ & $\omega_{\text {pum }}$ & $\omega_{\text {inst }}$ \\
\hline 100 & 0.006 & 6 \\
\hline
\end{tabular}

